Vol. 14, No 2, pp. 89-96, December 1995.

Universidad Católica del Norte

Antofagasta - Chile

\title{
ON COMMUTATIVITY OF RIGHT S-UNITAL RINGS WITH SOME POLYNOMIAL CONSTRAINTS
}

\author{
H. A. S. Abujabal and M. A. Khan \\ King Abdul Aziz University, Saudi Arabia
}

\begin{abstract}
We study the commutativity of certain class of rings, namely rings with unity 1 and right s-unital under each of the following properties $\left[y x^{m}-x^{n} f(y) x^{p}, x\right]=0,\left[y x^{m}+x^{n} f(y) x^{p}, x\right]=0$, where $f(t)$ is a polynomial in $t^{2} \mathbf{Z}[t]$ varying with pair of ring elements $x, y$ and $m, n, p$ are fixed non-negative integers. Moreover, the results have been extended to the case when $m$ and $n$ depend on the choice of $x$ and $y$ and the ring satisfies the Chacron's Theorem.
\end{abstract}




\section{Introduction.}

There is a multitude of conditions each of which implies the commutativity of certain rings. The equivalence of few such conditions to that of commutativity of rings was established by Komatsu and Tominaga [10] and Ashraf et al. [6]. The list of these equivalent conditions was further enlarged by authors jointly with Samman in [4]. The major purpose of this paper is to extend the work of Abujabal [5] and Ashraf et al. [7]. In fact, several commutativity theorems can be obtained as corollaries to our results, for instance $[1$, Theorem], [2, Theorem], [3, Theorem], [4, Theorem], [10, Theorem], [11, Theorem] and [12, Theorem].

Throughout this paper, $R$ will represent an associative ring (not necessarily with 1 ). Let $\mathbf{Z}[t]$ be the totality of polynomials in $t$ with coefficient in $\mathbf{Z}$ the ring of integers. As usual for any $x, y$ in $R$ the symbol $[x, y]$ will denote the commutator $x y-y z$. In Section 2, we investigate the commutativity of rings with unity 1 under the ring properties :

$\left(B_{1}\right)$ For every $x, y$ in $R$, there exists $f(x) \in X^{2} \mathbf{Z}[X]$, such that $\left[y x^{m}-x^{n} f(y) x^{p}, x\right]=0$, where $m, n$ and $p$ are fixed non-negative integers.

$\left(S_{1}\right)$ For every $x, y$ in $R$, there exists $f(X) \in X^{2} \mathbf{Z}[X]$, such that $\left[y x^{m}+x^{n} f(y) x^{p}, x\right]=0$, where $m, n$ and $p$ are fixed non-negative integers.

$\left(B_{2}\right)$ For every $x, y$ in $R$, there exist non-negative integers $m, n, p$ and $f(X) \in X^{2} \mathbf{Z}[X]$, such that $\left[y x^{m}+x^{n} f(y) x^{p}, x\right]=0$.

$\left(S_{2}\right)$ For every $x, y$ in $R$, there exist non-negative integers $m, n, p$ and $f(X) \in X^{2} \mathbf{Z}[X]$, such that $\left[y x^{m}+x^{n} f(y) x^{p}, x\right]=0$.

$(C A)$ For every $x, y$ in $R$, there exist $f(X), g(X)$ in $X^{2} \mathbf{Z}[X]$, such that $[x-f(x), y-g(y)]=0$.

The results of this paper are further extended to the right s-unital rings in the subsequent section 3 .

\section{Commutativity of rings with unity 1 .}

The following are the main results of this paper.

Theorem 1. Let $R$ be a ring with unity 1 . If $R$ is satisfying any one of the conditions $\left(B_{1}\right)$ and $\left(S_{1}\right)$, then $R$ is commutative (and conversely). 
Theorem 2. Let $R$ be a ring with unity 1 satisfying $(C A)$. If $R$ is satisfying any one of the conditions $\left(B_{2}\right)$ and $\left(S_{2}\right)$, then $R$ is commutative (and conversely).

In order to develop the proof of the above theorems first, we consider the following type of rings.

(1) $\left(\begin{array}{cc}G F(p) & G F(p) \\ 0 & G F(p)\end{array}\right), p$ a prime.

(1) $r\left(\begin{array}{cc}0 & G F(p) \\ 0 & G F(p)\end{array}\right), p$ a prime.

(1) $l\left(\begin{array}{cc}G F(p) & G F(p) \\ 0 & 0\end{array}\right), p$ a prime.

(2) $M_{\sigma}(F)=\left\{\left(\begin{array}{cc}a & b \\ 0 & \sigma(a)\end{array}\right) \mid a, b\right.$ in $\left.F\right\}$, where $F$ is a finite field with a non-trivial automorphism $\sigma$.

(3) A non-commutative division ring.

(4) $S=<1>+T, T$ as non-commutative radical subring of $S$.

(5) $S=<1>+T, T$ is a non-commutative subring of $S$ such that

$$
T[T, T]=[T, T] T=0 .
$$

In [14], Streb gave a nice classification for non-commutative rings, which yields a powerful tool in obtaining a number of commutativity theorems (cf. [1], [2], [3],[10] and [11]). It follows from the proof of [13, Corollary (1)], that if $R$ is a non-commutative ring with unity 1 , then there exists a factor subring of $R$ which is of type (1),(2),(3), (4) and (5). This observation gives the following result which plays the key role in our subsequent study.

Lemma 1. Let $P$ be a ring property which is inherited by factor subrings. If no rings of type $(1),(2),(3),(4)$ or (5) satisfy $P$, then every ring with unity 1 satisfying $P$ is commutative.

For the sake of convenience, we state the following well-known result due to Herstein [8].

Lemma 2. Let $R$ be a ring in which for every $x, y$ in $R$ there exists $f(x) \in X^{2} \mathbf{Z}[X]$, such that $[x-f(x), y]=0$. Then $R$ is commutative. 
Proof of Theorem 1. Let $R$ be a ring satisfying $\left(B_{1}\right)$. First, we shall show that no rings of type $(1),(2),(3),(4)$ or $(5)$ satisfies $\left(B_{1}\right)$. Let us begin with the ring of type (1). Then in $M_{2}(G F(p)), p$ a prime, we observe that $\left[e_{12} e_{12+22}^{m}-e_{12+22}^{n} f\left(e_{12}\right) e_{12+22}^{p}, e_{12+22}\right] \neq 0$ for all integers $m \geq 0, n \geq 0, p \geq 0$ and $f(X) \in X^{2} Z[X]$. Hence the ring of type (1) does not satisfy $\left(B_{1}\right)$.

Suppose that $B$ is the ring of type (2). Taking

$$
x=\left(\begin{array}{cc}
a & 0 \\
0 & \sigma(a)
\end{array}\right)(\sigma(a) \neq a), y=\left(\begin{array}{cc}
0 & 1 \\
0 & 0
\end{array}\right)
$$

in $\left(B=M_{\sigma}(F)\right)$.

One observes that

$$
\left[y x^{m}-x^{m} f(y) x^{p}, x\right]=-[x, y] x^{m}=(\sigma(a)-a)(\sigma(a))^{m} y \neq 0
$$

for all integers $m \geq 0, n \geq 0, p \geq 0$ and $f(X) \in X^{2} Z[X]$. Hence no rings of type (2) satisfy condition $\left(B_{1}\right)$.

Now, consider the ring $R$ of type (3). If $x$ is a unit of $R$, then for every $y$ in $R$ choose $f(x) \in X^{2} \mathbf{Z}[X]$, such that $\left[y x^{-m}-x^{-n} f(y) x^{-p}, x^{-1}\right]=0$. This implies that

$$
\left[y x^{-m}-x^{-n} f(y) x^{-p}, x\right]=0 .
$$

Thus it follows that

$$
x^{n}[x, y] x^{p}=[x, f(y)] x .
$$

Further, we consider $g(x) \in x^{2} \mathbf{Z}[x]$ such that $\left[f(y) x^{m}-x^{n} g(f(y)) x^{p}, x\right]=0$. This becomes

$$
x^{n}[x, g(f(y))] x^{p}=[x, f(y)] x^{m} .
$$

Combining (i) and (ii), we get $x^{n}[x, g(f(y))] x^{p}=x^{n}[x, y] x^{p}$.

But since $x$ is a unit, $[x, y-h(y)]=0$ for $g(f(y))=h(y) \in Y^{2} \mathbf{Z}[Y]$. Hence by Lemma $2, R$ is commutative which gives a contradiction. Thus a ring $R$ of type (3) does not satisfy $\left(B_{1}\right)$.

Suppose that $R$ has a factor subring of type (4). Let $a, b \in T$. Since $1-a$ is unit, using above paragraph there exists $f(X) \in X^{2} Z[X]$ such that $[a, b-f(b)]=-[1-a, b-f(b)]=0$. Thus $T$ is commutative by Lemma 2 . This is a contradiction, so $R$ does not satisfy a factor subring of type (4) which satisfy $\left(B_{1}\right)$. 
Finally, we consider $S=\langle 1\rangle+T$, where $T$ is a non-commutative subring of $S$ such that $T[T, T]=[T, T] T=0$. Let $R$ has a factor subring $S$. Choose $a, b \in T$ such that

$$
[a, b]=(a+1)^{n}[a, b](a+1)^{p}=[a, f(b)](a+1)^{m}=0 .
$$

This is a contradiction.

Hence, we have seen that no rings of Type $(1),(2),(3),(4)$ or (5) satisfy $\left(B_{1}\right)$. By Lemma $1, R$ is commutative.

Remark 1. If $R$ satisfies property $\left(S_{1}\right)$, then the proof will follow on the same lines as above.

Proof of Theorem 2. Suppose that $R$ satisfies property $\left(B_{2}\right)$. First, we assume that the rings of type (1). Then $\left[e_{12} e_{22}^{m}+e_{22}^{n} f\left(e_{12}\right) e_{22}^{p}, e_{22}\right] \neq 0$ for any integer $m \geq 0, n \geq 0, p \geq 0$, and $f(X) \in X^{2} \mathbf{Z}[X]$. This shows that no rings of type (1) satisfying $\left(B_{2}\right)$.

Using similar arguments as above one can prove that no rings of type (2) satisfy condition $\left(B_{2}\right)$. From this fact and Corollary 1 of [10], we obtain the required result.

Remark 2. The proof for the case $R$ satisfies $\left(S_{2}\right)$, will follow on the same lines as above.

Since there are non-commutative rings with $R^{2}$ being central, neither of these conditions guarantee the commutativity of arbitrary rings. However, we extend the study to the class of rings, which are called right s-unital. The results obtained here generalize $[1],[2],[3],[6],[9],[11]$.

\section{Extensions to right s-unital rings.}

An associative ring $R$ is called right (resp. left) s-unital if $x \in x R$ (resp. $x \in R x$ ) for each $x \in R$. Further $R$ is called s-unital if it is both right as well as left s-unital, i. e. $x \in R x \cap x R$ for each $x \in R$. 
If $R$ is an s-unital (resp. right or left s-unital) ring, then for any finite subset $A$ of $R$, there exists an element $e \in R$ such that $x e=e x=x$ (resp. $x e=x$ or $e x=x$ ) for all $x \in A$. Such an element $e$ is called the pseudo (left or pseudo right) identity of $\dot{A}$ in $R$. Before we go ahead with our task, we state the following lemma due to Komatsu, Nishinaka and Tominaga [11] in order to make our paper self- contained as far as possible.

Lemma 3. Let $R$ ve a right s-unital ring and not lef s-unital. Then $R$ has a factor sybring of type $(1)_{r}$.

If $R$ is a right s-unital ring satisfying $\left(B_{1}\right)$, then a careful scrutiny of the first paragraph of the proof of Theorem 1 shows that no rings of type $(1)_{\tau}$ satisfy $\left(B_{1}\right)$. By Lemma $3, R$ is s-unital and in view of Proposition 1 of [9], $R$ has unity 1. Thus $R$ is commutative by Theorem 1 , which gives the following.

Theorem 3. Let $R$ be a right s-unital ring stisfying any one of the conditions $\left(B_{1}\right)$ and $\left(S_{1}\right)$. Then $R$ is commutative (and conversely).

Corollary 1 . Let $m \geq 0, n \geq 0$ be fixed integers. Suppose that if $R$ is a right s-unital ring in which for every $x, y$ in $R$, there exists an integer $r=r(x, y)>1$ such that either $\left[y x^{m}-x^{n} y^{r} x^{p}, x\right]=0$ or $\left[y x^{m}+x^{n} y^{r} x^{p}, x\right]=0$. Then $R$ is commutative (and conversely).

From [10, Corollary 1], similarly we can prove the following result as follows :

Theorem 4. Let $R$ be a right s-unital ring satisfying $(C A)$. Suppose further that $R$ satisfies any one of the conditions $\left(B_{2}\right)$ and $\left(S_{2}\right)$. Then $R$ is commutative (and conversely).

Remark 3. One might conjecture a possible generalization of Theorem 1 and 2 when $R$ is left s-unital ring. The following example shows that there is a non-commutative left s-unital ring satisfying $\left(B_{1}\right)$ or $\left(S_{1}\right)$. 


\section{Example 1.}

Let $R=\left\{\alpha=\left(\begin{array}{ll}0 & 0 \\ 0 & 0\end{array}\right), \beta=\left(\begin{array}{ll}1 & 1 \\ 0 & 0\end{array}\right), \gamma=\left(\begin{array}{ll}0 & 0 \\ 1 & 1\end{array}\right), \delta=\left(\begin{array}{ll}1 & 1 \\ 1 & 1\end{array}\right)\right\}$ be a subring of $2 \times 2$ matrices over $G F(2)$. Then for any fixed positive integers $m, n, p$ and $f(x)$ in $X^{2} \mathbf{Z}[X], R$ satisfies $\left(B_{1}\right)$ or $\left(S_{1}\right)$. However, $R$ is not commutative, although $R$ is left s-unital.

Acknowledgments. The second author would like to thank professor Abdus Salam, the International Atomic Energy Agency and UNESCO for hospitaly at the International Center for Theoretical Physics, Trieste, Italy.

\section{REFERENCES}

[1] H. A. Abujabal and M. S. Khan : "On Commutativity Theorem for Rings", Internat. J. Math. and Math. Sci., 13, pp. 87 - 92, (1990).

[2] H. A. S. Abujabal : "Some Commutativity Results for Rings", Beiträge Zur Alg. und Geo., 32, pp. $141-151$, (1991).

[3] H. A. S. Abujabal and M. A. Khan: "On Commutativity of One-sided S-unital Rings", Internat J. Math. and Math. Sci., 15, pp. 813-818, (1992).

[4] H. A. S. Abujabal ; M. A. Khan and M. S. Samman : "On Commutativity of One Sided S-unital Rings", Tamkang J. Math., 23, pp. $253-267,(1992)$.

[5] H. A. S. Abujabal "Two Commutativity Results for Rings", Math. Japon, 38, pp. $297-299$, (1993).

[6] M. Ashraf ; M. A. Quadri and A. Ali : "On Commutativity of Onesided S-unital Rings", Rad. Mat., 6, pp. $111-117$, (1990).

[7] M. Ashraf ; M. A. Quadri and V. Jacob, "Commutativity of Right S-unital Rings Under Some Polynomial Constraints", Tamkang J. Math., 24, pp. $23-28$, (1993).

[8] I. N. Herstein : "Two Remarks on the Commutativity of Rings", Cand. J. Math., 7, pp. $411-412$, (1995). 
[9] Y. Hirano ; Y. Kobayashi and H. Tominaga : "Some Polynomial Identities and Commutativity of S-unital Rings", Math. J. Okayama Univ., 24, pp. $7-13,(1982)$.

[10] H. Komatsu and H. Tominaga : "Chacron's Condition and Commutativity Theorems", Math. J. Okayama Univ., 31, pp. $101-120$, (1989).

[11] H. Komatsu ; T. Nishinaka and H. Tominaga : "On Commutativity of Rings", Rad. Mat., 6, pp. $303-311$, (1990).

[12] T. Nishinaka : "A Commutativity Theorem for Rings", Rad. Mat., 6, pp. $357-359,(1990)$.

[13] W. Streb : "Zur Struktur Nichtommutativer Ringe", Math. J. Okayama Univ., 31, pp. 135 - 140, (1989).

Received : July 03, 1995.

H. A. S. Abujabal

M. A. Khan

Department of Mathematics

Faculty of Science

King Abdul Aziz University

P. O. Box 31464

Jeddah 21497

Saudi Arabia 